\title{
POSITRON ANNIHILATION STUDIES OF THE Ni-H SYSTEMS CONTAINING THERMALLY INDUCED CRYSTAL LATTICE DEFECTS
}

\author{
J. PAJĄK \\ Institute of Physics, Opole University, Oleska 48, 45-052 Opole, Poland
}

\begin{abstract}
The interaction between hydrogen and thermally induced defects has been studied in nickel samples using the positron annihilation technique. From measurements further evidence has been found for agglomeration of vacancies and small vacancy complexes into large-size three-dimensional void-type defects in the plastically deformed and electron beam irradiated samples containing hydrogen.
\end{abstract}

PACS numbers: 78.70.Bj

\section{Introduction}

In the previous papers $[1,2]$ the results of the investigations of positron annihilation quanta in plastically deformed and electron beam irradiated nickel samples saturated with hydrogen were reported. These results regarding the interaction of hydrogen with vacancies, voids and dislocations are rather surprising. Nickel belongs to the group of metals in which an intense trapping of positrons in crystal lattice defects is observed. This suggests that hydrogen should be also intensively trapped in these defects. However, no effect of hydrogen on positron annihilation, which could confirm this suggestion, has been observed. Our previous experiments do not confirm the suggestion regarding the hydrogen trapping at crystal lattice defects. The results indicate that introduction of hydrogen into nickel samples containing defects, created by plastic deformation or electron bombardment, causes the changes in the annihilation parameters suggesting an increase in both the concentration and dimensions of crystal lattice defects. It has been suggested that the main reason for the observed changes in the positron annihilation parameters is the agglomeration of defects in the presence of hydrogen atoms in the sample. Later measurements of the positron lifetime in plastically deformed samples have shown an increase in the intensity of the long living component in the samples containing hydrogen. The low value $(0.05 \mathrm{eV})$ of the hydrogen-vacancy and 
hydrogen-dislocation binding energy calculated in [3] seems to corroborate this suggestion.

In the present study the measurements of angular correlation curves, recovery processes and positron lifetime in nickel samples containing thermally induced crystal lattice defects, both hydrogen-free and saturated with hydrogen, were performed with the aim to find further evidences confirming the suggestions mentioned above.

\section{Experimental}

The samples with dimensions of $15 \times 10 \times 1 \mathrm{~mm}^{3}$ were prepared from nickel of $99.999 \%$ purity. Three kinds of samples were investigated:

1. Well-annealed ones containing only equilibrium defects obtained by high temperature tempering followed by slow cooling to room temperature (RT),

2. Defected samples tempered at $1670 \mathrm{~K}$ and quenched into liquid nitrogen. During the whole preparation process these samples were kept in a closed ampoule, at $10^{-6} \mathrm{Tr}$.

3. Samples prepared as in 2 , but additionally electrolytically charged with hydrogen up to the concentration of $2.5 \times 10^{-3}$ at. H/at.Ni in $5 \%$ water solution of $\mathrm{H}_{2} \mathrm{SO}_{4}$.

The angular correlation curves and isochronal annealing ones were measured with the aid of a standard spectrometer with a long-slit geometry.

The positron lifetime spectra were obtained at room temperature using a conventional fast-slow coincidence setup with $\mathrm{BaF}_{2}$ scintillators. The positron source $\left({ }^{22} \mathrm{Na}\right)$ were deposited on kapton foils of $4 \mathrm{mg} \mathrm{cm}^{-2}$ thickness. The source strength was about $30 \mathrm{mC}$. The time resolution of the spectrometer was $198 \mathrm{ps}$ (FWHM). The lifetime spectra were analyzed by means of the positron fit program.

\section{Results and discussion}

The results of the measurements of the angular correlation (AC) curves are presented in Figs. 1 and 2 in the form of relative difference curves. The shape of the curve in Fig. 1 confirms that an excess equilibrium concentration of defects has been created in the sample due to the quenching procedure.

In Fig. 2 the presence of hydrogen in the sample causes an increase in the curve in the angle interval ranging from 1 to 5 mrad. A similar result has been obtained previously for the samples defected by plastic deformation and by electron bombardment $[1,2]$. It is worth to mention, however, that there is no difference in the vicinity of $\Theta=0$.

From all the measured AC curves the $S, L$ and $R$ parameters [4] were determined. Their values are collected in Table $I$.

As it is known, the $S$ parameter depends on the defect type and concentration as well. The $L$ parameter depends on both concentration and dimensions of the defects, whereas the $R$ parameter depends on the defect size only. Although the difference between the $S$ and $L$ parameters for the hydrogen-free and hydrogen 


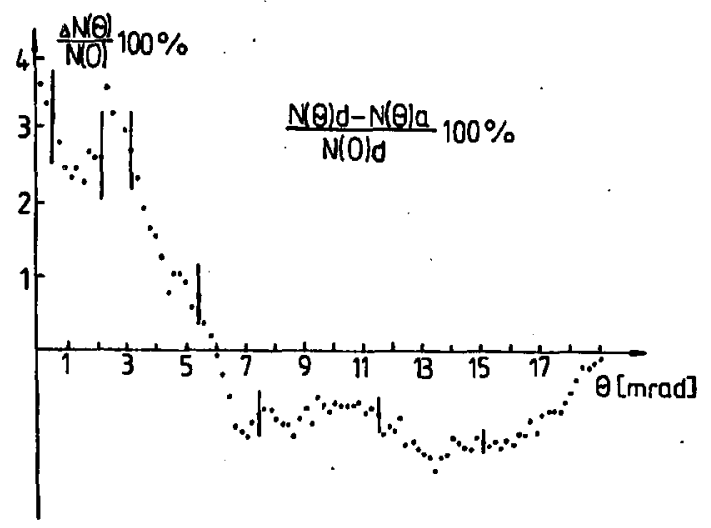

Fig. 1. Relative difference curve of the angular correlation curves for defected and well-annealed $\mathrm{Ni}$ samples.

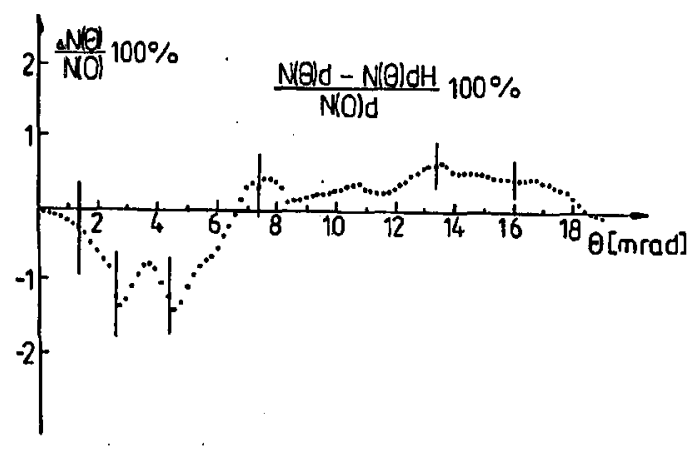

Fig. 2. Relative difference curve of the angular correlation curves for defected hydrogen-free and hydrogenated Ni samples.

TABLE I

Values of the $S, L$ and $R$ parameters as determined from the measured AC curves.

\begin{tabular}{l|c|c|c}
\hline \hline Sample & $S$ & $L$ & $R$ \\
\hline Well-annealed & 0.54 & 4.15 & - \\
Defected & 0.56 & 4.47 & 2.98 \\
Defected and hydrogenated & 0.57 & 4.58 & 3.38
\end{tabular}

containing defected nickel samples is small, the values for the defected sample are, as expected, the larger ones. The most striking result is, however, a large difference between the values of the $R$ parameter for hydrogen-free and hydrogenated defected nickel samples, suggesting that the dimensions of the defects in the sample containing hydrogen are much greater than those in hydrogen-free sample.

The presence of hydrogen in the sample containing defects produced by quenching must not change substantially the defect concentration. The interaction 
of hydrogen with already existing defects (vacancies) may lead to their agglomeration into voids. Hydrogen itself may cause a deformation of the sample thus leading to the creation of additional defects resulting in small changes in the $S$ and $L$ parameters as compared with appropriate values for the defected sample free of hydrogen.

The investigations of the recovery process of the hydrogen-free and hydrogenated defected nickel samples were performed in the temperature interval ranging from RT to $775 \mathrm{~K}$. The results for the hydrogen-free and hydrogenated nickel samples (both defected) are represented in Figs. 3a and b, respectively. As may be seen from Fig. 3a, a distinct recovery at $360 \mathrm{~K}$ occurs for the hydrogen-free sample. This is the well known stage III of the recovery process connected with annihilation of nonequilibrium vacancies [5]. The next stage of the recovery process occurring in the interval $675-773 \mathrm{~K}$ is connected with the recrystallization of the sample. From the recovery curve for the hydrogen containing sample (Fig. 3b) it follows that the stage III of the recovery process starts at $373 \mathrm{~K}$, and proceeds up to about $475 \mathrm{~K}$. This means that in the presence of hydrogen the vacancies become more stable and may result from vacancy migration and agglomeration in the presence of hydrogen into larger, three-dimensional clusters involving hydrogen atoms. Such clusters were already observed in quenched gold containing hydrogen [6]. The stage III of the recovery process in hydrogenated samples ends at $475 \mathrm{~K}$. At this temperature hydrogen becomes very mobile and its action of stabilizing the vacancy clusters is interrupted. This leads to the fast decay of the vacancy-type defects. Hydrogen, most probably, leaves the sample, and the curve becomes similar to that for the hydrogen-free sample.

The results of the positron lifetime measurements for the hydrogen-free and hydrogenated defected nickel samples are collected in Table II.

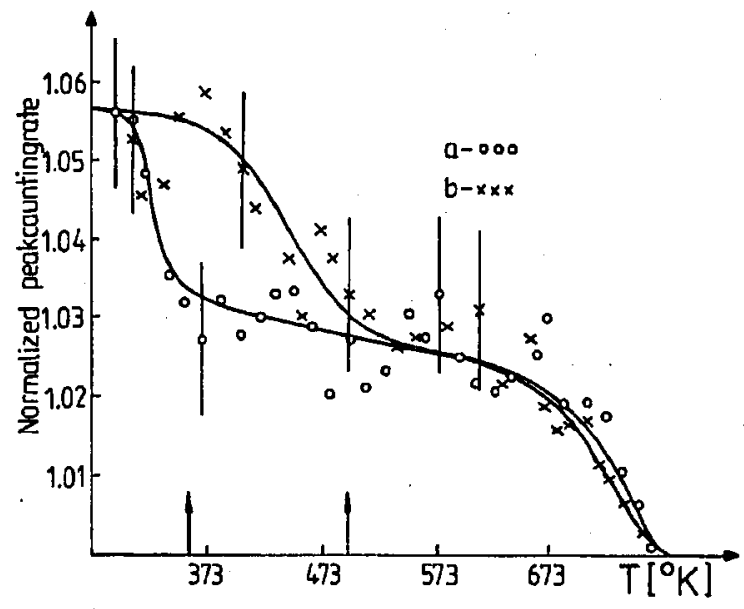

Fig. 3. The isochronal annealing curve for defected hydrogen-free $\mathrm{Ni}$ samples (a) and hydrogenated Ni sample (b). 
TABLE-II

Values of the positron lifetime for investigated nickel samples.

\begin{tabular}{l|c|c|c|c}
\hline \hline Sample & $\tau_{1}[\mathrm{ps}]$ & $\tau_{2}[\mathrm{ps}]$ & $I_{1}[\%]$ & $I_{2}[\%]$ \\
\hline Defected & $122 \pm 3$ & $288 \pm 6$ & 84.4 & 15.6 \\
Defected and hydrogenated & $124 \pm 3$ & $327 \pm 8$ & 87.9 & 12.1
\end{tabular}

The mean lifetime $\tau_{1}$ represents the averaged value of the bulk lifetime and the lifetime connected with trapping at vacancies or dislocations. Its value equals to $122 \mathrm{ps}$ being nearer to the bulk value $(110 \mathrm{ps})$ than to the value corresponding to trapping at vacancies or dislocations $(150 \mathrm{ps})$. This results from the small concentration of defects (4\%) created by quenching, which can be deduced from both the difference $\mathrm{AC}$ and recovery curves.

The component $\tau_{2}$ corresponds to the annihilation of positrons trapped at void-type defects. The introduction of hydrogen into sample does not substantially affect the value of $\tau_{1}$, but causes an increase in $\tau_{2}$ up to $327 \mathrm{ps}$. The most important reason for such an increase is the vacancy agglomeration, discussed earlier.

\section{Conclusions}

From the discussion of the results of the angular correlation and recovery curves as well as from the positron lifetime measurements performed it is concluded that all of them corroborate the previous suggestion [1,2] according to which the presence of hydrogen, in defected nickel samples, causes an agglomeration of vacancies or vacancy complexes into large-size three-dimensional void-type defects.

\section{References}

[1] J. Pajak, B. Rozenfeld, Surf. Coatings Technol. 28, 407 (1986).

[2] J. Pająk, B. Rozenfeld, in: Positron Annihilation, Eds. P.C. Jain, R.M. Singru, K.P. Gopinathan, World Scientific Publ. Co., Singapore 1985, p. 558.

[3] M.J. Baskes, C.F. Melius, Z. Phys. Chem. Neue Folge 116, 119 (1979).

[4] S. Mantl, W. Triftshauser, Phys. Rev. Lett. 34, 1554 (1975).

[5] Van den Beukel, in: Vacancies and Interstitials in Metals, Eds. A. Seeger, P. Schumacher, K. Schilling, J. Diehl, North-Holland, Amsterdam 1970, p. 427.

[6] J.A. Johnston, P.S. Dobson, R.E. Smallman, Proc. R. Soc. Lond. A 315, 231 (1970). 